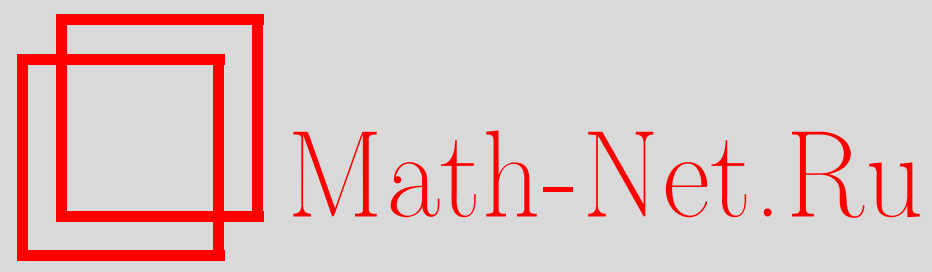

Д. В. Горбачев, Экстремальные задачи для целых функций экспоненциального сферического типа, Матем. заметки, 2000, том 68, выпуск 2, 179-187

DOI: https://doi.org/10.4213/mzm936

Использование Общероссийского математического портала Math-Net.Ru подразумевает, что вы прочитали и согласны с пользовательским соглашением http://www.mathnet.ru/rus/agreement

Параметры загрузки:

IP : 54.84 .234 .179

26 апреля 2023 г., $16: 26: 43$

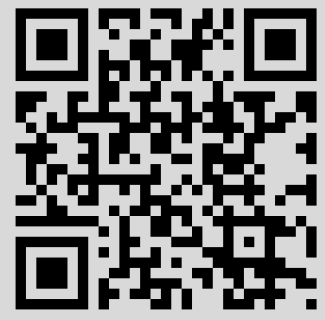




\section{ЭКСТРЕМАЛЬНЫЕ ЗАДАЧИ ДЛЯ ЦЕЛЫХ ФУНКЦИЙ ЭКСПОНЕНЦИАЛЬНОГО СФЕРИЧЕСКОГО ТИПА}

\section{Д. В. Горбачев}

Рассматриваются экстремальные задачи для целых функций экспоненциального сферического типа, связанные с важными экстремальными задачами об оптимальной точке (точке Черныха) в точном неравенстве Джексона в пространстве $L_{2}\left(\mathbb{R}^{n}\right)$ и связи между кодами и дизайнами на торе $T^{n}$.

Библиография: 17 названий.

Пусть $n \in \mathbb{N}, x, y \in \mathbb{R}^{n}, x y=x_{1} y_{1}+\cdots+x_{n} y_{n}$ - скалярное произведение векторов $x, y,|x|=\sqrt{x x}-$ длина вектора $x, B_{r}^{n}=\left\{x \in \mathbb{R}^{n}:|x| \leqslant r\right\}-$ шар в $\mathbb{R}^{n}$ радиуса $r>0 \mathrm{c}$ центром в нуле;

$$
L_{p}\left(\mathbb{R}^{n}\right)=\left\{f: \mathbb{R}^{n} \rightarrow \mathbb{C} \mid\|f\|_{p}=\left(\int_{\mathbb{R}^{n}}|f(x)|^{p} d x\right)^{1 / p}<\infty\right\} \quad(1 \leqslant p \leqslant \infty)
$$

для $\nu \in \mathbb{R}^{n}$

$$
\widehat{f}(\nu)=\int_{\mathbb{R}^{n}} f(x) e^{-i \nu x} d x
$$

- преобразование Фурье функции $f \in L_{1}\left(\mathbb{R}^{n}\right)[1],[2]$.

Пусть $E^{n R}$ - множество целых функций $f(z)\left(z \in \mathbb{C}^{n}\right)$ экспоненциального сферического типа, не превосходящего числа $R \geqslant 0$ [1, гл. 3]. Последнее означает, что

$$
\forall \varepsilon>0 \quad \exists A_{\varepsilon}>0 \quad \forall z \in \mathbb{C}^{n} \quad|f(z)| \leqslant A_{\varepsilon} e^{(R+\varepsilon)|z|} \quad\left(|z|=\sqrt{\left|z_{1}\right|^{2}+\cdots+\left|z_{n}\right|^{2}}\right) .
$$

При этом под типом функции $f(z)$ понимается точная нижняя грань чисел $d>0$, для которых выполняется неравенство $|f(z)| \leqslant A_{d} e^{d|z|}$ (для всех $z \in \mathbb{C}^{n}, A_{d}>0$ ).

Положим $E_{p}^{n R}=E^{n R} \cap L_{p}\left(\mathbb{R}^{n}\right)$. Известно [1, с. 120], что для любой функции $f \in E_{1}^{n R}$ $(R>0)$ преобразование Фурье $\widehat{f}(\nu)$ непрерьвно и имеет носитель в шаре $B_{R}^{n}(\operatorname{supp} \widehat{f} \subset$ $\left.B_{R}^{n}\right)$ и, более того, как следует, например, из [2, с. 19], $f(z)=(2 \pi)^{-n} \int_{B_{R}^{n}} \widehat{f}(\nu) e^{i z \nu} d \nu$.

Данная статья посвящена решению двух экстремальных задач для целых функций из $E_{1}^{n R}$.

Работа выполнена при финансовой поддержке Российского фонда фундаментальных исследований, грант № 97-01-00318, и Минобразования, грант № 97-0-1.4-78. 
ЗАДАчА 1. Пусть $R>0$. Обозначим

$$
\begin{gathered}
F^{n R}=\left\{f \in E_{1}^{n R}:\left.f(x)\right|_{x \in \mathbb{R}^{n}} \in \mathbb{R}, \widehat{f}(0)=\int_{\mathbb{R}^{n}} f(x) d x \geqslant 0\right\}, \\
\lambda(f)=\sup \left\{|x|: x \in \mathbb{R}^{n}, f(x)>0\right\} \quad\left(f \in F^{n R}\right) .
\end{gathered}
$$

Найти величину

$$
\Lambda_{n}(R)=\Lambda\left(F^{n R}\right)=\inf \left\{\lambda(f): f \in F^{n R}, f \not \equiv 0\right\}
$$

При $n=1$ эта задача была поставлена (в несколько иной форме) и решена Логаном [3] $\left(\Lambda_{1}(R)=\pi / R\right)$. Этот же результат вытекает из более ранних работ Черныха [4], [5]. Величина $\Lambda_{3}(R)=2 \pi / R$ найдена Московским [6]. При $n>1$ в случае функций экспоненциального типа $(R, \ldots, R) \in \mathbb{R}_{+}^{n}$ (т.е. удовлетворяющих условию $|f(z)| \leqslant A_{\varepsilon} e^{(R+\varepsilon)\left(\left|z_{1}\right|+\cdots+\left|z_{n}\right|\right)}, \operatorname{supp} \widehat{f} \subset[-R, R]^{n}[1$, гл. 3]) точное решение аналогичной задачи (равное $\pi \sqrt{n} / R$ ) получено Бердьшевой [7].

Пусть $\alpha=(n-2) / 2, j_{\alpha}(u)=2^{\alpha} \Gamma(\alpha+1) u^{-\alpha} J_{\alpha}(u)$ - нормированная функция Бесселя $\left(j_{\alpha}(0)=1\right), J_{\alpha}(u)-$ функция Бесселя [8, гл. 7]. Обозначим через $0<q_{\alpha 1}<q_{\alpha 2}<\cdots$ положительные нули $J_{\alpha}(u)$.

Tеорема 1. Если $n \in \mathbb{N}, \alpha=(n-2) / 2, R>0, m o$

$$
\Lambda_{n}(R)=\frac{2 q_{\alpha 1}}{R} \text {. }
$$

Экстремальной функиией является функиия Черньха-Юдина

$$
F_{R}(x)=\frac{j_{\alpha}^{2}(R|x| / 2)}{1-R^{2}|x|^{2} /\left(2 q_{\alpha 1}\right)^{2}} \in F^{n R} .
$$

Доказательство будет приведено ниже.

Задача 1 оказалась тесно связанной с важной экстремальной задачей об оптимальной точке в точном неравенстве Джексона в пространстве $L_{2}\left(\mathbb{R}^{n}\right)$. Пусть $f \in L_{2}\left(\mathbb{R}^{n}\right)$,

$$
E_{R}\left(f, \mathbb{R}^{n}\right)_{2}=\inf _{g \in E_{2}^{n R}}\|f-g\|_{2}
$$

- величина наилучшего приближения порядка $R>0$ функции $f(x)$; для $\delta>0$ обозначим

$$
\omega\left(\delta, f, \mathbb{R}^{n}\right)_{2}=\sup _{|h| \leqslant \delta}\|f(x+h)-f(x)\|_{2}
$$

- модуль непрерывности функции $f(x)$,

$$
D\left(\delta, R, \mathbb{R}^{n}\right)_{2}=\sup _{\substack{f \in L_{2}\left(\mathbb{R}^{n}\right) \\ f \not 0}} \frac{E_{R}\left(f, \mathbb{R}^{n}\right)_{2}}{\omega\left(\delta, f, \mathbb{R}^{n}\right)_{2}}
$$

- точная константа Джексона. Тогда [6], [9] $D\left(\delta, R, \mathbb{R}^{n}\right)_{2}$ невозрастает по $\delta$ и $D\left(\delta, R, \mathbb{R}^{n}\right)_{2}$ $\geqslant 1 / \sqrt{2}$ для всех $\delta, R>0$, причем $D\left(2 q_{\alpha 1} / R, R, \mathbb{R}^{n}\right)_{2}=1 / \sqrt{2}, D\left(q_{\alpha 1} / R, R, \mathbb{R}^{n}\right)_{2}>1 / \sqrt{2}$. Точка

$$
\tau_{n}(R)=\inf \left\{\delta>0: D\left(\delta, R, \mathbb{R}^{n}\right)_{2}=\frac{1}{\sqrt{2}}\right\}
$$

назьвается оптимальной точкой в точном неравенстве Джексона или точкой Черныха. Бердьшева показала [7], что $\tau_{n}(R)=\Lambda\left(F^{n R}\right)$. Таким образом, из теоремы 1 следует, что

$$
\tau_{n}(R)=\frac{2 q_{\alpha 1}}{R}
$$


ЗАДАчА 2. Пусть $R>0$. Обозначим

$$
\begin{gathered}
G^{n R}=\left\{f \in E_{1}^{n R}:\left.f(x)\right|_{x \in \mathbb{R}^{n}} \in \mathbb{R}, \widehat{f}(0)=0, \widehat{f}(\nu) \geqslant 0 \quad\left(\nu \in \mathbb{R}^{n}\right)\right\}, \\
\sigma(f)=\sup \left\{|x|: x \in \mathbb{R}^{n}, f(x)<0\right\}=\lambda(-f) \quad\left(f \in G^{n R}\right) .
\end{gathered}
$$

Найти величину

$$
\Sigma_{n}(R)=\Sigma\left(G^{n R}\right)=\inf \left\{\sigma(f): f \in G^{n R}, f \not \equiv 0\right\}
$$

Эта задача возникла под влиянием работы Юдина [10], где установлена связь между кодами и дизайнами на торе $T^{n}=[-\pi, \pi)^{n}$ (здесь несколько изменены обозначения [10]).

ОПРЕДЕЛЕНИЕ. Конечное множество $W \subset T^{n}$ называется:

1) $\delta$-кодом, если

$$
d(W)=\min \left\{|x-y|_{T^{n}}: x, y \in W, x \neq y\right\}=\delta \quad(\delta \in(0, \pi \sqrt{n}]),
$$

где $|x-y|_{T^{n}}=\min _{\nu \in \mathbb{Z}^{n}}|x-y+2 \pi \nu|-$ метрика на торе;

2) шаровым дизайном порядка $R=R(W)$, если $R>0$ - наибольшее число, при котором кубатурная формула

$$
\frac{1}{(2 \pi)^{n}} \int_{T^{n}} t(x) d x=\frac{1}{|W|} \sum_{u \in W} t(u)
$$

точна для любого тригонометрического полинома

$$
t(x)=\sum_{\substack{\nu \in \mathbb{Z}^{n} \\|\nu|<R}} c_{\nu} e^{i \nu x}
$$

со спектром в шаре $B_{R}^{n}$.

Основным результатом [10] является оценка

$$
d(W) R(W) \leqslant 2 q_{\alpha 2} \quad\left(\forall W \subset T^{n}, R(W)>\frac{2 q_{\alpha 2}}{\pi \sqrt{n}}\right) .
$$

Она получена на пути решения экстремальной задачи построения тригонометрического полинома $t(x)$ со спектром в $B_{R}^{n}$, неотрицательного при $|x| \geqslant \delta\left(x \in T^{n}\right)$, у которого $c_{0}=0, c_{\nu} \geqslant 0$ и $\delta$-наименьшее. Эта задача трудная (и не решена), поэтому в [10] строится тригонометрический полином с “достаточно мальм" $\delta$. Для этого по сути решается задача 2 , т. е. ищется функция $f(x)$ из $G^{n R}$ (в [10] функция $f \in L_{1}\left(\mathbb{R}^{n}\right)$, supp $\widehat{f} \subset B_{R}^{n}$, $\widehat{f}(0)=0, \widehat{f} \geqslant 0)$, которая неотрицательна при $|x| \geqslant \delta\left(x \in \mathbb{R}^{n}\right)$ с наименьшим $\delta$. После этого в качестве "хорошего" тригонометрического полинома $t(x)$ используется периодизация $f(x)$, т. е. $t(x)=\sum_{\nu \in \mathbb{Z}^{n}} f(x+2 \pi \nu)$. В [10] предлагается функция

$$
G_{R}(x)=\frac{j_{\alpha}^{2}(R|x| / 2)}{\left(1-R^{2}|x|^{2} /\left(2 q_{\alpha 1}\right)^{2}\right)\left(1-R^{2}|x|^{2} /\left(2 q_{\alpha 2}\right)^{2}\right)} \in L_{1}\left(\mathbb{R}^{n}\right)
$$

с $\delta=2 q_{\alpha 2} / R$ (отсюда как раз следует оценка $\left.(1)\right)$, для которой установлено, что $\operatorname{supp} \widehat{G}_{R}$ $\subset B_{R}^{n}, \widehat{G}_{R}(0)=0, \widehat{G}_{R} \geqslant 0$, т. е. $G_{R} \in G^{n R}$. При этом известно [11], [10], что в одномерном случае $\delta$, равное $3 \pi / R$ на всем классе $G^{1 R}$, уменьшить нельзя, т. е. по сути решена задача 2 и $\Sigma_{1}(R)=3 \pi / R$. Следующий результат показывает, что и при $n>1$ величину $\delta$ равную $2 q_{\alpha 2} / R$ уменьшить нельзя и $G_{R}(x)$ - наилучшая (экстремальная) функция. 
Tеорема 2. Eсли $n \in \mathbb{N}, \alpha=(n-2) / 2, R>0, m o$

$$
\Sigma_{n}(R)=\frac{2 q_{\alpha 2}}{R}
$$

Экстремальной функцией является функция Юдина

$$
G_{R}(x)=\frac{j_{\alpha}^{2}(R|x| / 2)}{\left(1-R^{2}|x|^{2} /\left(2 q_{\alpha 1}\right)^{2}\right)\left(1-R^{2}|x|^{2} /\left(2 q_{\alpha 2}\right)^{2}\right)} \in G^{n R} .
$$

Доказательство будет приведено ниже.

Нам понадобится одна теорема из [12] (см. также [13]), представляющая собой квадратурную формулу Гаусса на полуоси.

Пусть $E_{0}^{\delta}-$ множество целых четных функций $\varphi(u)$ одной комплексной переменной $u \in \mathbb{C}$ экспоненциального типа, не превосходящего числа $\delta \geqslant 0[1$, гл. 3$]$, т. е. $E_{0}^{\delta}=\{\varphi \in$ $\left.E^{1 \delta}: \varphi(-u)=\varphi(u)\right\}$.

Теорема 3 [12]. Если $\alpha>-1, \tau>0$, mо для всех $\varphi \in E_{0}^{2 \tau}$ maкux, что

$$
\int_{0}^{\infty}|\varphi(t)| t^{2 \alpha+1} d t<\infty
$$

справедлива квадратурная формула

$$
\int_{0}^{\infty} \varphi(t) t^{2 \alpha+1} d t=\frac{1}{\tau^{2 \alpha+2}} \sum_{k=1}^{\infty} \gamma_{\alpha k} \varphi\left(\frac{q_{\alpha k}}{\tau}\right)
$$

$2 \partial e$

$$
\gamma_{\alpha k}=\frac{2 q_{\alpha k}^{2 \alpha}}{J_{\alpha+1}^{2}\left(q_{\alpha k}\right)}>0
$$

Пусть далее $n \in \mathbb{N}, \alpha=(n-2) / 2($ так что $2 \alpha+1=n-1), q_{\alpha k}=q_{k}, \gamma_{\alpha k}=\gamma_{k}$, $S^{n-1}=\left\{x \in \mathbb{R}^{n}:|x|=1\right\}$ - единичная сфера в $\mathbb{R}^{n}$ с центром в нуле, $\omega_{n-1}=\left|S^{n-1}\right|=$ $2 \pi^{n / 2} /(\Gamma(n / 2))$ - площадь $S^{n-1}[2$, с. 16$]$. Также нам потребуется одна лемма.

Лемма. Пусть $f \in E_{1}^{n \delta}$. Тогда функиия

$$
\varphi(u)=\int_{S^{n-1}} f(u \xi) d \xi \quad(u \in \mathbb{C})
$$

являющаяся усреднением функции $f(x)$ по сферам, удовлетворяет условиям:

1) $\varphi \in E_{0}^{\delta}$

2) $\int_{0}^{\infty}|\varphi(t)| t^{2 \alpha+1} d t \leqslant\|f\|_{1}<\infty$;

3) $\int_{0}^{\infty} \varphi(t) j_{\alpha}(s t) t^{2 \alpha+1} d t=\omega_{n-1}^{-1} \int_{S^{n-1}} \widehat{f}(s \nu) d \nu(s \in \mathbb{R})$. 
ДоказАтельСтво. 1) Разложим целую функцию $f(z)$ в ряд Тейлора $[1$, гл. 3$]$

$$
f(z)=\sum_{\nu \in \mathbb{Z}_{+}^{n}} f_{\nu} z^{\nu} \quad\left(z \in \mathbb{C}^{n}, z^{\nu}=z_{1}^{\nu_{1}} \cdots z_{n}^{\nu_{n}}\right)
$$

абсолютно сходящийся для всех $z$. Тогда для произвольного $u \in \mathbb{C}$

$$
\varphi(u)=\sum_{\nu \in \mathbb{Z}_{+}^{n}} f_{\nu} u^{\nu_{1}+\cdots+\nu_{n}} \int_{S^{n-1}} \xi_{1}^{\nu_{1}} \cdots \xi_{n}^{\nu_{n}} d \xi .
$$

Поскольку величина $A_{\nu}=\int_{S^{n-1}} \xi_{1}^{\nu_{1}} \cdots \xi_{n}^{\nu_{n}} d \xi$ равна нулю, если хотя бы одна из компонент вектора $\nu$ нечетна и $\left|A_{\nu}\right| \leqslant \omega_{n-1}$, то функцию $\varphi(u)$ можно представить в виде ряда $\sum_{k=0}^{\infty} a_{k} u^{2 k}$, очевидно, абсолютно сходящегося для любого $u$. Отсюда следует, что $\varphi(u)$ является целой четной функцией. То, что она имеет экспоненциальньй тип $\leqslant \delta$ вытекает из того, что $f \in E^{n \delta}$, и оценки

$$
|\varphi(u)| \leqslant \int_{S^{n-1}}|f(u \xi)| d \xi \leqslant \int_{S^{n-1}} A_{\varepsilon} e^{(\delta+\varepsilon)|u \xi|} d \xi=\omega_{n-1} A_{\varepsilon} e^{(\delta+\varepsilon)|u|}
$$

Таким образом, $\varphi \in E_{0}^{\delta}$.

2) Воспользуемся формулой интегрирования в $\mathbb{R}^{n}[2$, с. 46$]$

$$
\int_{\mathbb{R}^{n}} g(x) d x=\int_{0}^{\infty} \int_{S^{n-1}} g(t \xi) d \xi t^{n-1} d t
$$

и (2). Тогда

$$
\int_{0}^{\infty}|\varphi(t)| t^{2 \alpha+1} d t \leqslant \int_{0}^{\infty} \int_{S^{n-1}}|f(t \xi)| d \xi t^{n-1} d t=\int_{\mathbb{R}^{n}}|f(x)| d x=\|f\|_{1}<\infty .
$$

3) Учитывая, что $\|f\|_{1}<\infty$, имеем для $s \in \mathbb{R}$ :

$$
\int_{S^{n-1}} \widehat{f}(s \nu) d \nu=\int_{S^{n-1}} \int_{\mathbb{R}^{n}} f(x) e^{-i s \nu x} d x d \nu=\int_{\mathbb{R}^{n}} f(x) \int_{S^{n-1}} e^{-i s \nu x} d \nu d x .
$$

Известно [2, с. 176], что

$$
\int_{S^{n-1}} e^{i s \nu x} d \nu=\omega_{n-1} j_{\alpha}(s|x|) .
$$

Отсюда с помощью (2) и (3) получаем, что

$$
\begin{aligned}
\int_{S^{n-1}} \widehat{f}(s \nu) d \nu & =\int_{\mathbb{R}^{n}} f(x) \omega_{n-1} j_{\alpha}(s|x|) d x=\int_{0}^{\infty} \int_{S^{n-1}} f(t \xi) \omega_{n-1} j_{\alpha}(s t) d \xi t^{n-1} d t \\
& =\omega_{n-1} \int_{0}^{\infty} \varphi(t) j_{\alpha}(s t) t^{2 \alpha+1} d t .
\end{aligned}
$$

Отметим, что последний интеграл сходится равномерно по $s \in \mathbb{R}$, так как $\left|j_{\alpha}(s t)\right| \leqslant 1$ для $t \in \mathbb{R}($ см. [8, с. 23] или формулу (4)). Лемма доказана. 
ДоКАЗАТЕЛЬСТво ТЕОРЕМЫ 1. Отметим, что для всех $\delta, R>0$ вьполнено равенство

$$
\Lambda_{n}(\delta R)=\frac{1}{\delta} \Lambda_{n}(R)
$$

что сразу следует из очевидного равенства $\lambda(f(\delta x))=\lambda(f(x)) / \delta$. Поэтому достаточно доказать, что $\Lambda_{n}(2)=q_{1}$, откуда

$$
\Lambda_{n}(R)=\frac{2}{R} \Lambda_{n}(2)=\frac{2 q_{1}}{R} .
$$

Покажем, что для всех $\delta \in(0,2)$ вьполнена оценка $\Lambda_{n}(\delta) \geqslant q_{1}$. Предположим обратное, т.е. $\Lambda_{n}(\delta)<q_{1}$. Это означает, что существует функция $f \in F^{n \delta}$, для которой $\lambda(f)<q_{1}$.

Рассмотрим усреднение функции $f(x)$ по сфере

$$
\varphi(u)=\int_{S^{n-1}} f(u \xi) d \xi \quad(u \in \mathbb{C}) .
$$

Так как для $t>0, \xi \in S^{n-1}$ выполнено неравенство $f(t \xi) \leqslant 0$ при $|t \xi|=t \geqslant \lambda(f)$, то, очевидно, и $\varphi(t) \leqslant 0$ при $t \geqslant \lambda(f)$.

В силу леммы $\varphi \in E_{0}^{\delta}, \int_{0}^{\infty}|\varphi(t)| t^{2 \alpha+1} d t<\infty$ и

$$
\int_{0}^{\infty} \varphi(t) t^{2 \alpha+1} d t=\widehat{f}(0) \geqslant 0
$$

(последнее соотношение получено из пункта 3 ) леммы при $s=0$ ). Отсюда, в частности, следует, что функция $\varphi \in E_{0}^{\delta} \subset E_{0}^{2}$ полностью удовлетворяет условиям теоремы 3. Применяя эту теорему к ней как к функции экспоненциального типа меньшего 2 (т.е. с $\tau=1)$, получим

$$
0 \leqslant \int_{0}^{\infty} \varphi(t) t^{2 \alpha+1} d t=\sum_{k=1}^{\infty} \gamma_{k} \varphi\left(q_{k}\right) .
$$

Но $\varphi(t) \leqslant 0$ при $t \geqslant \lambda(f)$, а $\lambda(f)<q_{1}$. Поэтому (5) возможно лишь в случае, когда $\int_{0}^{\infty} \varphi(t) t^{2 \alpha+1} d t=\widehat{f}(0)=0$ и целая четная функция $\varphi(u)$ имеет в точках $q_{k}$ по крайней мере двойные нули.

Известно [8, с. 72, 90], что нормированная функция Бесселя $j_{\alpha}(u)$ является целой четной функцией экспоненциального типа 1 и имеет разложение Вейерштрасса

$$
j_{\alpha}(u)=\prod_{k=1}^{\infty}\left(1-\frac{u^{2}}{q_{k}^{2}}\right) .
$$

Таким образом, функцию $\varphi(u)$ можно представить в виде [14]

$$
\varphi(u)=j_{\alpha}^{2}(u) \psi(u) \quad(\psi \not \equiv 0-\text { целая функция })
$$

что невозможно, так как слева стоит целая функция экспоненциального типа $\delta<2$, а справа - типа $\geqslant 2$. Тем самым мы пришли к противоречию. 
Итак, для всех $\delta \in(0,2)$

$$
\Lambda_{n}(2)=\frac{\delta}{2} \Lambda_{n}(\delta) \geqslant \frac{\delta}{2} q_{1},
$$

откуда при $\delta \rightarrow 2$ получаем $\Lambda_{n}(2) \geqslant q_{1}$.

Для доказательства оценки сверху $\Lambda_{n}(2) \leqslant q_{1}$ достаточно взять функцию Черныха-Юдина

$$
F_{2}(x)=\frac{j_{\alpha}^{2}(|x|)}{1-|x|^{2} / q_{1}^{2}} \quad\left(x \in \mathbb{R}^{n}\right),
$$

являющуюся преобразованием Фурье весовой функции Черныха-Юдина, построенной при $n=1$ Черныхом [4], а при $n>1$ - Юдиным [15] и используемой (после периодизации) при доказательстве точного неравенства Джексона в пространстве $L_{2}\left(T^{n}\right)$. Функция $F_{2} \in E^{n 2}$, поскольку $j_{\alpha}^{2}(u) /\left(1-u^{2} / q_{1}^{2}\right) \in E_{0}^{2}$ (см. также [1, с. 135]), $\left\|F_{2}\right\|_{1}<\infty$ и

$$
\int_{\mathbb{R}^{n}} F_{2}(x) d x=\omega_{n-1} \int_{0}^{\infty} \frac{j_{\alpha}^{2}(t)}{1-t^{2} / q_{1}^{2}} t^{2 \alpha+1} d t=\omega_{n-1} \sum_{k=2}^{\infty} \gamma_{k} \frac{j_{\alpha}^{2}\left(q_{k}\right)}{1-q_{k}^{2} / q_{1}^{2}}=0
$$

Таким образом, функция $F_{2}(x)$ принадлежит классу $F^{n 2}$. Отметим, что вид этой функции угадьвается из (5). Действительно, легко предположить из (5), что экстремальная функция должна получаться из целой четной функции, имеющей в точке $q_{1}$ простой нуль, а в точках $q_{k}(k \geqslant 2)$ двойные нули, т. е. как раз

$$
\left(1-\frac{u^{2}}{q_{1}^{2}}\right) \prod_{k=2}^{\infty}\left(1-\frac{u^{2}}{q_{k}^{2}}\right)^{2}=\frac{j_{\alpha}^{2}(u)}{1-u^{2} / q_{1}^{2}}
$$

Ясно, что экстремальной функцией для величины $\Lambda_{n}(R)=2 q_{1} / R(R>0)$ является функция

$$
F_{R}(x)=F_{2}\left(\frac{R}{2} x\right)=\frac{j_{\alpha}^{2}(R|x| / 2)}{1-R^{2}|x|^{2} /\left(2 q_{1}\right)^{2}} \in F^{n R} .
$$

Теорема доказана.

ДОКАЗАТЕЛЬСТВО ТЕОРЕМЫ 2. Будем постоянно использовать факты, установленные при доказательстве теоремы 1 . Аналогично соотношению для величины $\Lambda_{n}(\delta R)$ для любых $\delta, R>0$ вьполнено равенство

$$
\Sigma_{n}(\delta R)=\frac{1}{\delta} \Sigma_{n}(R)
$$

поэтому достаточно доказать, что $\Sigma_{n}(2)=q_{2}$, откуда

$$
\Sigma_{n}(R)=\frac{2}{R} \Sigma_{n}(2)=\frac{2 q_{2}}{R} .
$$

Покажем, что для всех $\delta \in(0,2) \Sigma_{n}(\delta) \geqslant q_{2}$. Предположим обратное, т. е. $\Sigma_{n}(\delta)<q_{2}$. Это означает, что существует $f \in G^{n \delta}$, для которой $\sigma(f)<q_{2}$.

Как и при доказательстве теоремы 1 , рассмотрим усреднение функции $f(x)$

$$
\varphi(u)=\int_{S^{n-1}} f(u \xi) d \xi \quad(u \in \mathbb{C}) .
$$


Так как для $t>0, \xi \in S^{n-1}$ вьполнено неравенство $f(t \xi) \geqslant 0$ при $|t \xi|=t \geqslant \sigma(f)$, то, очевидно, и $\varphi(t) \geqslant 0$ при $t \geqslant \sigma(f)$.

Для функции $\varphi(u)$ выполняются условия:

$$
\varphi \in E_{0}^{\delta}, \quad \int_{0}^{\infty}|\varphi(t)| t^{2 \alpha+1} d t<\infty, \quad \int_{0}^{\infty} \varphi(t) t^{2 \alpha+1} d t=\widehat{f}(0)=0
$$

а также из пункта 3) леммы для всех $\varepsilon>0$

$$
\int_{0}^{\infty} \psi_{\varepsilon}(t) \varphi(t) t^{2 \alpha+1} d t=-\frac{1}{\omega_{n-1}} \int_{S^{n-1}} \widehat{f}(\varepsilon \nu) d \nu \leqslant 0,
$$

где $\psi_{\varepsilon}(u)=j_{\alpha}(\varepsilon \sigma(f))-j_{\alpha}(\varepsilon u) \in E_{0}^{\varepsilon},\left|\psi_{\varepsilon}(t)\right| \leqslant 2(t \in \mathbb{R})$.

Далее воспользуемся идеей доказательства теоремы 3 из [16]. Возможны 3 случая: 1) $\left.\sigma(f) \leqslant q_{1} ; 2\right) \sigma(f) \in\left(q_{1}, q_{2}\right)$ и $\left.\varphi\left(q_{1}\right) \geqslant 0 ; 3\right) \sigma(f) \in\left(q_{1}, q_{2}\right)$ и $\varphi\left(q_{1}\right)<0$.

По теореме 3 для $\varphi \in E_{0}^{\delta} \subset E_{0}^{2}$ вьполнено равенство

$$
0=\int_{0}^{\infty} \varphi(t) t^{2 \alpha+1} d t=\sum_{k=1}^{\infty} \gamma_{k} \varphi\left(q_{k}\right)
$$

Но $\varphi(t) \geqslant 0$ при $t \geqslant \sigma(f)$ и $\varphi\left(q_{1}\right) \geqslant 0$ для случаев 1$)$ и 2$)$. Следовательно, целая четная функция $\varphi(u)$ имеет в точках $q_{k}$ нули, причем в точках $q_{k}(k \geqslant 2)$, по крайней мере, двойные. Отсюда функцию $\varphi(u)$ можно представить в виде

$$
\varphi(u)=\frac{j_{\alpha}^{2}(u)}{1-u^{2} / q_{1}^{2}} \psi(u) \quad(\psi \not \equiv 0-\text { целая функция })
$$

что невозможно, так как слева стоит целая функция экспоненциального типа $\delta<2$, а справа - типа $\geqslant 2$.

В случае 3) применим теорему 3 к функции $\psi_{\varepsilon} \varphi \in E_{0}^{\delta+\varepsilon} \subset E_{0}^{2}(\varepsilon<2-\delta)$ :

$$
0 \geqslant \int_{0}^{\infty} \psi_{\varepsilon}(t) \varphi(t) t^{2 \alpha+1} d t=\sum_{k=1}^{\infty} \gamma_{k} \psi_{\varepsilon}\left(q_{k}\right) \varphi\left(q_{k}\right)
$$

Известно [8], что функция $j_{\alpha}(t)$ убывает при $0 \leqslant t \leqslant q_{\alpha+1,1}$ и $\left|j_{\alpha}(t)\right| \leqslant\left|j_{\alpha}\left(q_{\alpha+1,1}\right)\right|<1$ при $t \geqslant q_{\alpha+1,1}, n>1$ (последнее вытекает из монотонного убывания последовательных максимумов функции $\left|j_{\alpha}(t)\right|$, равных $\left.\left|j_{\alpha}\left(q_{\alpha+1, k}\right)\right|[17]\right)$. Отсюда при достаточно малом $\varepsilon$ следует, что $\psi_{\varepsilon}\left(q_{1}\right)<0$ и $\psi_{\varepsilon}\left(q_{k}\right)>0$ при $k \geqslant 2$. Но $\varphi(t) \geqslant 0$ при $t \geqslant \sigma(f)$, откуда для выбранного $\varepsilon \psi_{\varepsilon}\left(q_{k}\right) \varphi\left(q_{k}\right) \geqslant 0$ при $k \geqslant 2$ и $\psi_{\varepsilon}\left(q_{1}\right) \varphi\left(q_{1}\right)>0$, что в силу $(7)$ невозможно. Во всех трех случаях пришли к противоречию.

Итак, для всех $\delta \in(0,2)$ вьполнено неравенство

$$
\Sigma_{n}(2)=\frac{\delta}{2} \Sigma_{n}(\delta) \geqslant \frac{\delta}{2} q_{2},
$$

откуда при $\delta \rightarrow 2$ получаем $\Sigma_{n}(2) \geqslant q_{2}$.

Формулы (6) и (7) подсказьвают, что в качестве экстремальной функции для оценки $\Sigma_{n}(2) \leqslant q_{2}$ можно попытаться взять функцию, получаемую из целой четной функции с 
простьми нулями в точках $q_{1}, q_{2}$ и двойными нулями в точках $q_{k}(k \geqslant 3)$, т. е. функцию Юдина

$G_{2}(x)=\left(1-\frac{|x|^{2}}{q_{1}^{2}}\right)\left(1-\frac{|x|^{2}}{q_{2}^{2}}\right) \prod_{k=3}^{\infty}\left(1-\frac{|x|^{2}}{q_{k}^{2}}\right)^{2}=\frac{j_{\alpha}^{2}(|x|)}{\left(1-|x|^{2} / q_{1}^{2}\right)\left(1-|x|^{2} / q_{2}^{2}\right)} \quad\left(x \in \mathbb{R}^{n}\right)$,

которая, как уже было отмечено, принадлежит классу $G^{n 2}$.

Для величины $\Sigma_{n}(R)=2 q_{2} / R(R>0)$ экстремальной функцией является функция $G_{R}(x)=G_{2}(R x / 2) \in G^{n R}$. Теорема доказана.

\section{СПИСОК ЦИТИРОВАННОЙ ЛИТЕРАТУРЫ}

[1] Никольский C.M. Приближение функций многих переменных и теоремы вложения. М.: Наука, 1977.

[2] Стейн И., Вейс Г. Введение в гармонический анализ на евклидовых пространствах. М.: Мир, 1974.

[3] Logan B. F. Extremal problems for positive-definite bandlimited functions. II. Eventually negative functions // SIAM J. Math. Anal. 1983. V. 14. № 2. P. 253-257.

[4] Черных Н. И. О неравенстве Джексона в $L_{2} / /$ Тр. МИАН. 1967. Т. 88. С. 71-74.

[5] Chernykh N. I., Arestov V. V. On the $L_{2}$-approximation of periodic functions by trigonometric polynomials // Approximation and Functions Spaces. Proc. Inter. Conf. (Gdansk, 1979). Amsterdam: North-Holland, 1981. P. 25-43.

[6] Московский А. В. Теоремы Джексона в пространствах $L_{p}\left(\mathbb{R}^{n}\right)$ и $L_{p, \lambda}\left(\mathbb{R}_{+}\right) / /$Изв. ТулГУ. Сер. матем., мех., информатика. 1997. Т. 3. №1. С. 44-70.

[7] Бердышева Е. Е. Две взаимосвязанные экстремальные задачи для целых функций многих переменных // Матем. заметки. 1999. Т. 66. №3. С. 336-350.

[8] Бейтмен Г., Эрдейи А. Высшие трансцендентные функции. Т. 2. М.: Наука, 1966.

[9] Бабенко А.Г. Точное неравенство Джексона-Стечкина в пространстве $L^{2}\left(\mathbb{R}^{m}\right) / / \mathrm{Tp}$. ИММ УрО РАН. 1998. Т. 5. С. 182-198.

[10] Юдин В.А. Расположение точек на торе и экстремальные свойства полиномов // Тр. МИАН. 1997. Т. 219. С. 453-463.

[11] Logan B. F. Extremal problems for positive-definite bandlimited functions. I. Eventually positive functions with zero integral functions // SIAM J. Math. Anal. 1983. V. 14. № 2. P. 253-257.

[12] Grorev G. R., Rahman Q. I. A quadrature formulae with zeros of Bessel functions as nodes // Math. Comput. 1995. V. 64. P. 715-725.

[13] Chanem R. B. Explicit quadrature formulae for entire functions of exponential type // J. Approximation Theory. 1998. V. 92. P. 267-279.

[14] Маркушевич А. И. Теория аналитических функций. Т. 2. М.: Наука, 1968.

[15] Юдин В. А. Многомерная теорема Джексона в $L_{2} / /$ Матем. заметки. 1981. Т. 29. № 2. C. $309-315$.

[16] Юдин В. А. Код и дизайн // Дискретная матем. 1997. Т. 9. № 2. С. 3-11.

[17] Иванов В. И. О приближении функций в пространствах $L_{p} / /$ Матем. заметки. 1994. Т. 56. № 2. C. $15-40$. 